\title{
Deciding the Effective Position of Fuel Injector using Cfd.
}

\author{
Shinde Vijay S, Kothmire Pramod P.
}

\begin{abstract}
We are going to test the fluid flow of fuel injector by the process of computational fluid dynamics (CFD). Fluid separator is a mechanical device which helps two or more fluid to separate from a mixture. Fluid separator has its application in many pharmaceutical and automobile industries. Fluid separator had made the most promising device in the industry. Most of the modification has been made in the design of the fuel injector with respect to geometry and thermal design. In a CFD, the examination of fluid flow in accordance with its physical properties such as velocity, temperature, density and viscosity are obtained. The study shows the best performance of fuel injector with respect to shape and flow rates. The purpose of this report is to corelate various design of injector in the industry. In this Paper we can find out the effective position of the fuel injector using CFD.
\end{abstract}

Keywords: Chemical Industries, Fluid separator, Multi-fluid chemical inlet, Physical Properties,

\section{INTRODUCTION}

The Fluid centrifuge transmits input take a look at pressure to the output finish across Associate in Nursing elastomeric membrane. The input finish of the fluid centrifuge may be connected to a gas pressure supply, or it may be crammed with fluid and connected to hydraulic pressure supply. The term centrifuge in oil field nomenclature designates a pressure vessel used for separating well fluids created from oil and gas wells into vaporous and liquid parts. A centrifuge for crude oil production could be a massive vessel designed to separate production fluids into their constituent parts of oil, gas and water. A separating vessel is also brought up within the following ways: Oil and gas centrifuge, Separator, Stage centrifuge, Trap, Knockout vessel (Knockout drum, knockout lure, water knockout, or liquid knockout), Flash chamber (flash vessel or flash trap), enlargement centrifuge or enlargement vessel, Scrubber (gas scrubber), Filter (gas filter). These separating vessels are commonly used on a manufacturing lease or platform close to the wellhead, manifold, or tank battery to separate fluids created from oil and gas wells into oil and gas or liquid and Emulsions are caused once oil associate degreed water are forced to combine within the presence of an emulsion-stabilizing wetting agent.

Revised Manuscript Received on October 30, 2019

* Correspondence Author

Mr. Vijay Subhash Shinde, student of Masters of Technology in Heat Power from School of Mechanical and Civil Engineering, MIT Academy of Engineering Alandi (D), Savitribai Phule Pune University, Pune, India,

Mr. Pramod P Kothmire, Assistant Professor in School of Mechanical and Civil Engineering MIT Academy of Engineering Alandi (D), Savitribai Phule Pune University, Pune, India,

(C) The Authors. Published by Blue Eyes Intelligence Engineering and Sciences Publication (BEIESP). This is an open access article under the CC BY-NC-ND license (http://creativecommons.org/licenses/by-nc-nd/4.0/)
Emulsifying agents are inherent in created oil and gas condensation, yet as several chemical treatments, like film-forming corrosion inhibitors. These emulsion-stabilizing surfactants collect at the boundary or interface between the oil and water and create a robust, resilient film that resists breaking. This film prevents the distributed droplets from coalescing into larger droplets and rift of the mixture. Halliburton offers customized emulsion breakers for each water-in-oil (regular) and oil-in-water (reverse) emulsions, serving to improve oil-water separations whereas serving to maintain sales contract specifications and increasing oil and gas production rates.

\section{LITERATURE REVIEW}

Sengar, J.A.M. Kuipers, R.A. van Santen, J.T. Padding [1]. The approach is then accustomed analyze a reaction (a) following the Langmuir-Hinshelwood dynamics, (b) generating product particles with completely different self diffusivity values as compared to the chemical particles. The model developed will more incorporate reactions occurring within advanced geometries (pore diffusion) and even be accustomed study advanced reaction systems that the mean-field assumption isn't any longer valid. 2018 printed by Elsevier. the power to model physical systems occurring at multiple time scales, with the present procedure power at hand, has been extensively accustomed resolve or discover new phenomena in the main within the field of fluid and solid mechanics. the entire runtime of a simulation is $t \frac{1}{4}$ tstart $p$ treactor $\mathrm{p}$ teq. $\mathrm{Lz}=\mathrm{u}$, streaming, collision, reflection and reaction occur. For teq, the stabilization time of the catalyst, once more all the processes occur. For a linear method, teq $1 / 4$ zero and therefore the simulation time typically scales as a result of reflections. we are able to conjointly assume tstart. treactor. For dekaliter 1/4 10; picture element 1/4 100; Re 1/4 twenty-one, the simulation time was around $4000 \mathrm{~s}$ [1] Christophe Vallée, Thomas Höhne, Horst-Michael Prasser, Tobias Sühnel [2]. The aim of the numerical simulations is that the validation of prediction of the slug flow with the prevailing point flow models in-built the industrial code ANSYS CFX. more it's of interest to prove the understanding of the final fluid dynamic mechanism resulting in slug flow and to spot the essential parameters touching the most slug flow parameters. CFD pre-test calculations were done to point out the likelihood of slug flow generation during a real pure mathematics and at relevant parameters for reactor safety. The simulation was performed on a flat model representing the hot-leg of the German Konvoi-reactor at the dimensions of $1: 3$. The fluids were water and steam at a pressure of fifty bar and therefore the corresponding saturation temperature of $263.9^{\circ} \mathrm{C}$. 
The results of the CFD-calculation show initial Associate in Nursing upwards inclination of the stagnant water in direction of the steam generator, driven by the momentum exchange from the injected steam. Further, waves ar generated at random within the horizontal a part of the hot-leg and grow to slugs within the region of the bend [2].

A. Efendioglu, J. Mendez \& H. Turkoglu [1]. The main sizes of a apparatus were determined for a particular oil gas mixture victimization the empirical correlations from API and literature. to research the consequences of the position of the diverter and perforated plates on the separation efficiency; two-phase flow simulations were conducted victimization CFD package ANSYS CFX. The CFD simulations were administrated for 2 completely different diverter plate distances from the body of water. The diverter plate was situated a hundred millimeter and a hundred and seventy millimeters from the body of water. in addition, simulation was conjointly performed with perforated baffle plates once the diverter plate is positioned a hundred and seventy millimeters from the body of water. To estimate the consequences of those completely different configurations on separation potency, the CFD simulations results were compared. it's been ascertained that perforated plates and position. it's been ascertained that perforated plates and position of the body of water diverter have an effect on the separation potency. once the body of water diverter is found at a hundred millimeter from the body of water, the separation potency of ninety-eight. 5 which there's obtained. once the body of water diverter is found at a distance of a hundred and seventy millimeter from the body of water, the separation potency will increase to ninety-nine.32\%. once perforated plates are assembled to the apparatus, the separation potency will increase more. Finally, this study has incontestable that the interior style of oil-gas separators is very important, as a result of even a really little increase in separation potency could save loads of cash. This analysis continues to be beneath development, it's planned to gift a benchmark between the sphere info and therefore the CFD simulations, so as to perform a sensitivity analysis and assess the dependence of the most properties, models, interpolation schemes, mesh, analysis kind and its results [4].

A. H. BROWN AND C. HANSON [5]. A study was product of the consequences of high energy oscillatory electrical fields on the conjugation of liquid drops at a flat liquid-liquid interface. Drop volumes of zero.002-0.008 cm3 and frequencies of 1-10 kc/sec were used, the most applied potential being around ten $\mathrm{kV}$ r.m.s. The mean rest time of droplets at the interface was measured, and therefore the effects of temperature, frequency and strength were evaluated; the utilization of electrical suggests that to help subsiding in mixer-settlers nine seems to possess a minimum of 2 benefits over a d.c. source. Electrolysis of the binary compound section is greatly reduced and therefore the current necessities are abundant less. Electrical strategies of aiding subsiding seem solely to be effective for the water-in-oil variety of emulsion. We believe, however, that forced mechanical vibrations and cavitation inside the drop are answerable for the premature rupture of the film and therefore the succeeding conjugation. the foremost vital results are those of table three that show that the sphere within the drop, instead of the charge it carries, is answerable for premature conjugation [5].
Matthew Lawa, Poh Seng Leea, Ming Hanb, NingpingChenb, Lijun Liub [6]. fluid flow in several manifold configurations ar numerically investigated victimization 3-D procedure Fluid Dynamics (CFD) simulations to gauge the uniformity of the flow distribution and therefore the pressure come by every style. The cases are simulated with 5 body of water flow rates, and therefore the performance of the various manifolds are assessed. the foremost effective style can then be fictional and more valid with experiments. Three-dimensional numerical simulations are performed within the gift study to research the flow distribution of 3 manifold styles. From the simulation results, it will be finished that:

A. The generic style of the manifold, DO isn't favorable in terms of flow distribution and pressure drop. this can be severally because of the momentum of the flow that carries the fluid pass the primary four shops and therefore the presence of recirculation zones.

B. Flow distribution and pressure drop will be improved victimization easy nevertheless effective modifications via the shifting of the body of water and shops positions, as seen in D1 and D2.

C. D1 and D2 have additional uniform flow distribution throughout every of their ten shops compared to try and do with their higher flow uniformity index.

D. D1 and D2 sustained lower pressure drop compared to try and do for the complete vary of body of water flow rates.

\section{DESIGN METHDOLOGY}

\section{A. Geometry modelling}

A 3D geometric CATIA model having Cylindrical vessel dimensions Length $320 \mathrm{~cm}$ and diameter $140 \mathrm{~cm}$ was used for the analysis. Case- 1 consist of a geometry fuel injector are in upper region and the holes are front and back. In Case-2 I can only rotate the fuel injector by 180 degree or holes are in top and bottom of the fuel injector. In Case- 3 distributer are in lower region and the holes are front and back as compared to last case the position of the fuel injector rotate by180 degree or holes are top and bottom position of the fuel injector. As shown in the fig 1 below:

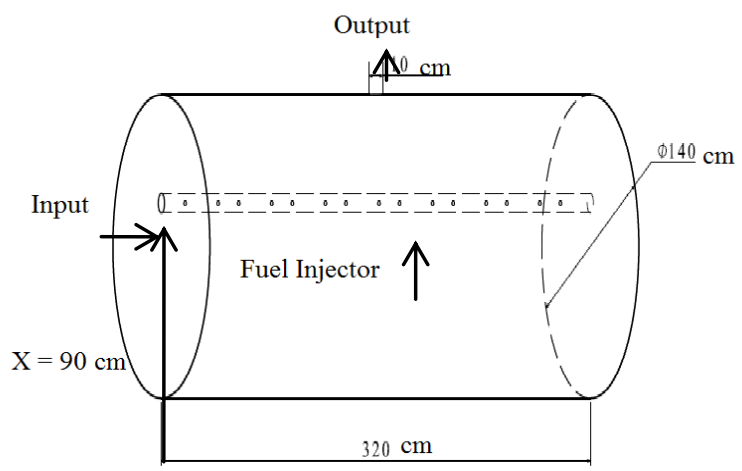

Fig 1: Geometry was simulated by running 4 cases,

Case $1, x=90 \mathrm{~cm}$ (Orientation of nozzle normal to the surface),

Case 2, $x=90 \mathrm{~cm}$ (Orientation of nozzle perpendicular to surface), 
Case $3, x=30 \mathrm{~cm}$ (Orientation of nozzle normal to the surface),

Case $4, x=30 \mathrm{~cm}$ (Orientation of nozzle perpendicular to surface).

Where,

$\mathrm{X}=$ Distance of fuel injector form bottom surface of vessel.

A geometrical model of a fuel injector design or problem statement is as shown in above fig.2. The main vessel (separator) having 5 litres capacity. Required separation time for this process is 1 minutes. To determine fluid separator parameters at first, we have decided to study fuel injector design which helps to overcome the arising problems like a meshing of a complicated geometry, number of iterations in FLUENT. Horizontal pipe of the fuel injector contains 15 number of segregated holes. Where, on one side 8 holes $(1,3,5,7,9,11,13$ and 15$)$ are located at $40 \mathrm{~cm}$ distance from the centre and on another side, 7 holes $(2,4,6,8,10,12$ and 14) are located at $20 \mathrm{~cm}$ distance from the centre.

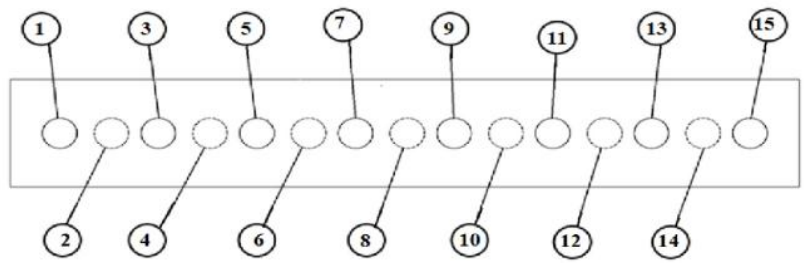

Fig.2: Fuel injector with holes in segregated form.

\section{B. Meshing}

For 3D modelling here we used AutoCAD and CATIA V5 respectively. From fig.7. we can observe that water enters at inlet and a stream of water split through holes of the distributor. The fine mesh generation was created on an assembly with 123352 nodes and 114342 elements. It was performed by ANSYS workbench as shown in fig.3. Fluent is used as solver. Tetrahedral mesh is used for this geometry.

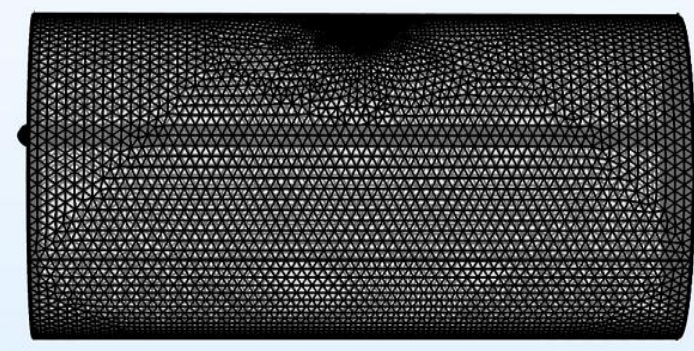

Fig 7. Geometry Meshing

\section{Material properties}

Following are the properties of the materials consisting in the cylindrical Vessel. After few research surveys the most appropriate was selected based on its density and viscosity. The unit of properties are in S.I system.

Table1. Materials and their properties.

\begin{tabular}{|c|c|c|c|c|}
\hline Material & Type & $\begin{array}{c}\text { Chemical } \\
\text { Formula }\end{array}$ & $\begin{array}{c}\text { Density } \\
(\mathrm{Kg} / \mathrm{m} 3)\end{array}$ & $\begin{array}{c}\text { Viscosity } \\
(\mathrm{Kg} / \mathrm{m}-\mathrm{s})\end{array}$ \\
\hline
\end{tabular}

\begin{tabular}{|c|c|c|c|c|}
\hline Water & Fluid & H2O & 998.2 & 0.001003 \\
\hline Aluminum & Solid & AL & 2719 & ----- \\
\hline
\end{tabular}

D. Governing Equations and Boundary Conditions

The fluid continuity equation, accounting for flow, can be written as:

$$
\frac{\partial\left(\rho_{f} u\right)}{\partial x}+\frac{\partial\left(\rho_{f} v\right)}{\partial y}+\frac{\partial\left(\rho_{f} w\right)}{\partial z}=0
$$

The momentum equations in $\mathrm{x}, \mathrm{y}$ and $\mathrm{z}$ direction, Accounting for momentum can be written as:

$$
\begin{aligned}
& u \frac{\partial\left(\rho_{f} u\right)}{\partial x}+v \frac{\partial\left(\rho_{f} u\right)}{\partial y}+w \frac{\partial\left(\rho_{f} u\right)}{\partial z}=\frac{\partial\left(\mu_{f} \frac{d u}{d x}\right)}{\partial x}+\frac{\partial\left(\mu_{f} \frac{d u}{d y}\right)}{\partial y}+\frac{\partial\left(\mu_{f} \frac{d u}{d z}\right)}{\partial z}-\frac{\partial p}{\partial x} \\
& u \frac{\partial\left(\rho_{f} v\right)}{\partial x}+v \frac{\partial\left(\rho_{f} v\right)}{\partial y}+w \frac{\partial\left(\rho_{f} v\right)}{\partial z}=\frac{\partial\left(\mu_{f} \frac{d v}{d x}\right)}{\partial x}+\frac{\partial\left(\mu_{f} \frac{d v}{\partial y}\right)}{\partial y}+\frac{\partial\left(\mu_{f} \frac{d v}{d z}\right)}{\partial z}-\frac{\partial p}{\partial y} \\
& u \frac{\partial\left(\rho_{f} w\right)}{\partial x}+v \frac{\partial\left(\rho_{f} w\right)}{\partial y}+w \frac{\partial\left(\rho_{f} w\right)}{\partial z}=\frac{\partial\left(\mu_{f} \frac{d w}{d x}\right)}{\partial x}+\frac{\partial\left(\mu_{f} \frac{\partial w}{\partial y}\right)}{\partial y}+\frac{\partial\left(\mu_{f} \frac{d w}{d z}\right)}{\partial z}-\frac{\partial p}{\partial z}
\end{aligned}
$$

There were two zones air and water out of which fluid zones. For these fluid zones we have considered outlet as pressure outlet and inlet as a velocity inlet.

Table 2. Parameter for Estimation of values.

\begin{tabular}{|c|c|}
\hline Parameters & Values \\
\hline $\begin{array}{c}\text { Volume Capacity of } \\
\text { Separator (V) }\end{array}$ & 5 Liters \\
\hline Volumetric Flow Rate (Q) & 5 LPM \\
\hline Diameter of Inlet Pipe (D1) & $10 \mathrm{~cm}$ \\
\hline
\end{tabular}

From Table 2, we can easily calculate the value for flow velocity at effective position of distributors.

The Boundary condition for inlet pipe (d1) is velocity and at outlets is gauge pressure, normal to boundary. After satisfaction of this condition, we can find out velocity and pressure value through output holes by changing position of inlet feeder for different cases. From continuity equation (1), we can find out inlet pipe (d1) velocity.

$$
\mathrm{Q}=\mathrm{A}^{*} \mathrm{~V}
$$

Where,

$\mathrm{Q}=$ Volume of flow rate,

$\mathrm{A}=$ Cross sectional area of input pipe, $\mathrm{V}=$ Velocity of flow.

\section{PRAPOSED SYSTEM}

The methodology adopted for present work is explained below,

To study the Position of the holes: -

To study the Previous Position of the holes in fluid separator. Observe the Position of the holes i.e. number of holes, position, and angle etc. 


\section{Experimental validation: -}

Finding out the stress and deformation with an analytically on Position of fluid separator. This value is validated with previous position of the separator.

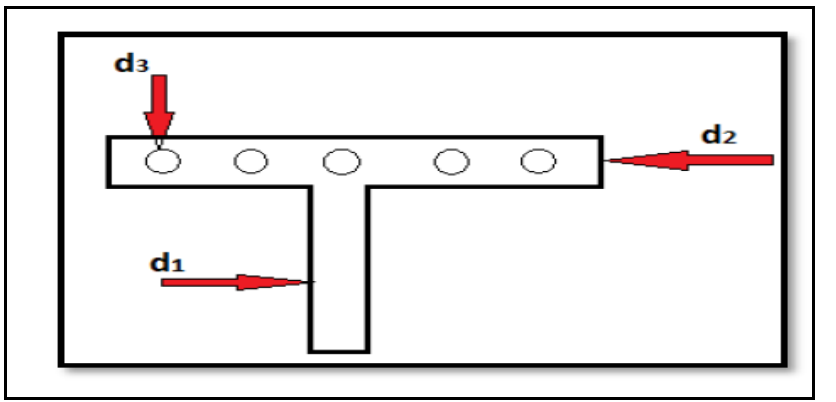

Fig.3 Previous Holes Position in the Fluid Flow Separation

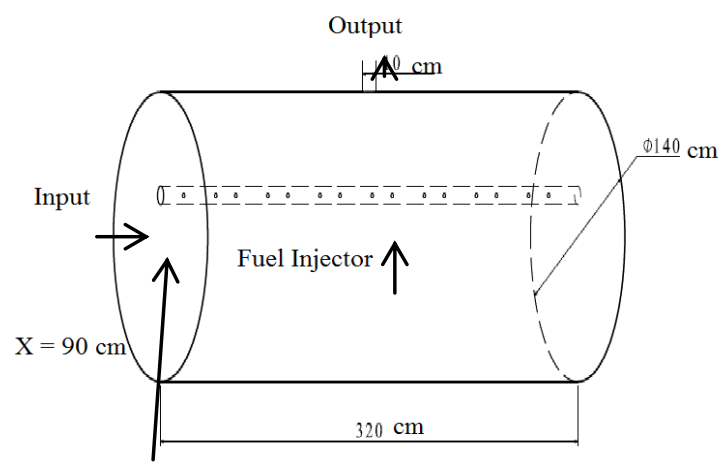

Fig. 4 Modified Holes Position in The Fluid Flow Separator

This is modified holes position in the fluid flow separator. It consists of the Input, Output and Distributer. If input is Water (H2o) at 5 LPM to the distributer and we can calculate the time, velocity at the outlet, and particle track.

\section{ANALYSIS REPORT}

Analysis report only shows the result of Case- 1 because this is the best case of my research work it takes a longer time in fluid separator. The quality of separator achieved maximum efficiency so that's why I can only shows the result. of case1.
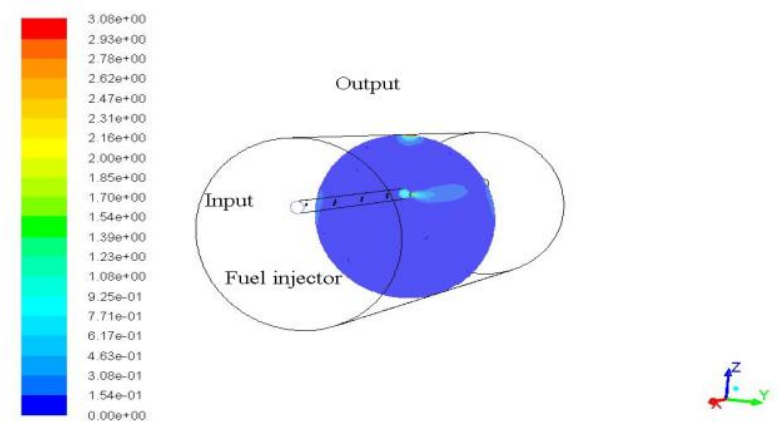

Fig.5 Counters of velocity magnitudes along $\mathrm{X}$-axis.

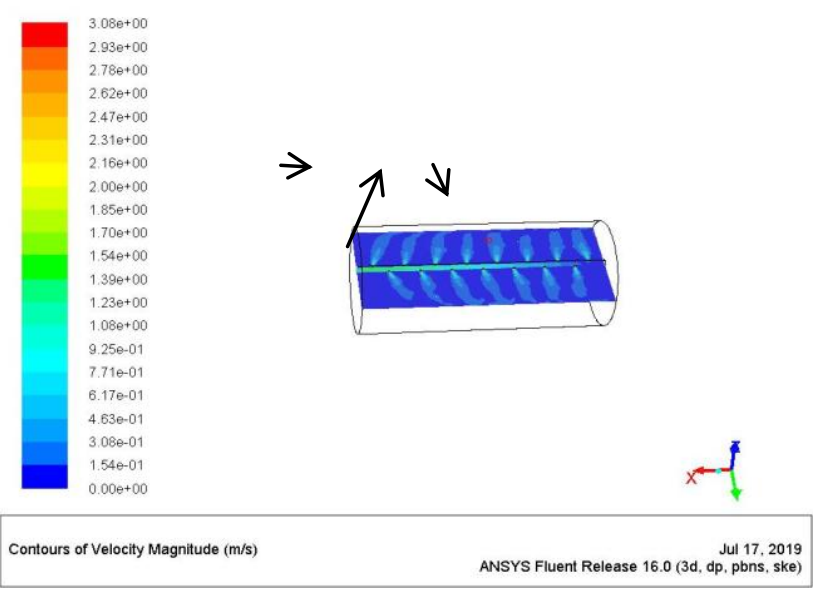

Fig.6 Counters of velocity magnitudes along Z-axis.

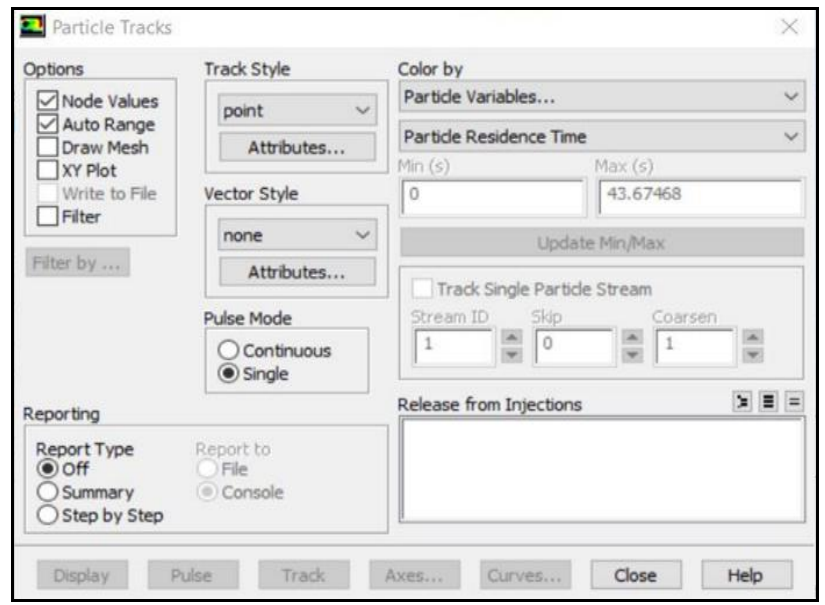

Fig.7Practical Tracks

Fig 7 shows the particle track I can send input as water and collected at output of the domain and this test will require large amount of time $43.67 \mathrm{sec}$ that time is maximum in across all the cases.

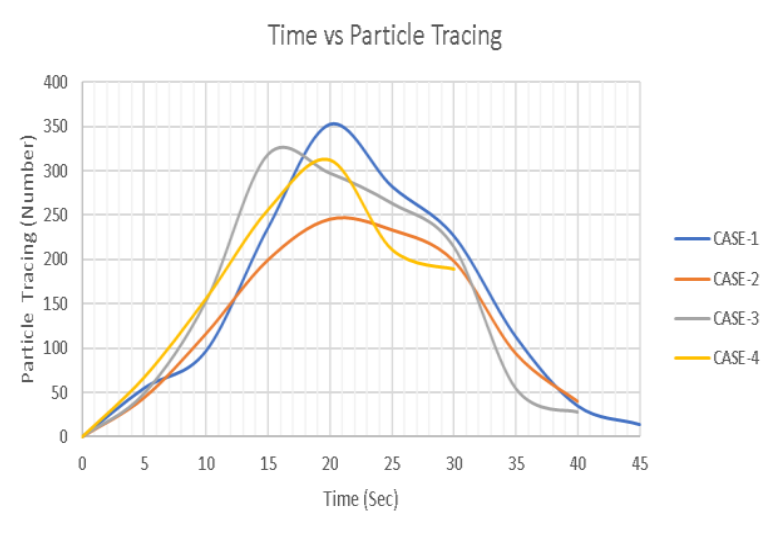

Fig.8 Graph of all Cases

Fig 8. This graph shows the combination of all the four cases that have been study. On the $\mathrm{X}$-axis represents the time to be taken by each case and on $\mathrm{Y}$-axis shows particle tracing (Number) i.e. collected at the outlet of the domain in each case 5 sec time interval. 


\section{CONCLUSION}

Fluid flow analysis of fluid separator by CFD analysis process. The experimental result shows that the flow rate and the time taken by the separator for better functionality changes with respect to time. The separator along with the increased holes and angles brought a significant change in the quality delivery in output. The quality of separator output has maximum efficiency when it takes a longer time in fluid separator. Quality of separator remains low when the separator takes a less time for the delivery of output. Most of the modifications has been done in the holes and the angels. No deformation has been seen in the separator.

\section{ACKNOWLEDGMENT}

It is optional. The preferred spelling of the word "acknowledgment" in American English is without an "e" after the "g." Use the singular heading even if you have many acknowledgments. Avoid expressions such as "One of us (S.B.A.) would like to thank ... ." Instead, write "F. A. Author thanks" Sponsor and financial support acknowledgments are placed in the unnumbered footnote on the first page.

\section{REFERENCES}

1. 1.Allahyarov, E., Gompper, G., 2002. Mesoscopic solvent simulations: multiparticlecollision dynamics of three-dimensional flows. Phys. Rev. E - Statis. Nonlinear Soft Matter Phys. 66 (3), 1-9.

2. 2.Alves, M.A., Delgado, J.M., Guedes De Carvalho, J.R., 2006. Mass transfer from cylinders and plane surfaces buried in packed beds in alignment with the flow direction. Chem. Eng. Sci. 61 (4), 1174-1183.

3. 3.Berendsen, H.J.C., 1991. Transport properties computed by linear response through weak coupling to a bath. Comput. Simul. Mater. Sci., 139-155 Chen, S., Doolen, G.D., 1998. Lattice Boltzmann method for fluid flows. Annu. Rev. Fluid Mech. 30 (1), 329-364.

4. 4.Churchill, S.W., 1988. Viscous Flows: The Practical Use of Theory. Butterworths. Collins, F.C., Kimball, G.E., 1949. Diffusion-controlled reaction rates. J. Colloid Sci. 4 (4), 425-437.

5. 5.Delgado, J.M.P.Q., 2006. A critical review of dispersion in packed beds. Heat Mass Transf. 42 (4), 279-310. Deprez, L., de Buyl, P., 2017. Passive and active colloidal chemotaxis in a microfluidic channel: mesoscopic and stochastic models. Soft Matter 13 (19), 3532-3543.

6. 6.Eigenberger, G., 2008. Catalytic fixed-bed reactors. In: Handbook of Heterogeneous Catalysis. Wiley-VCH Verlag GmbH \& Co., KGaA Weinheim, Germany, pp. 2075-2106.

7. 7.Karabey, A., Numerical Analysis of Gas-Solid Particule Two Phase Flow for Size Classification of Particules, Ph.D. Thesis, Gazi University, Turkey, 2001.

\section{AUTHORS PROFILE}

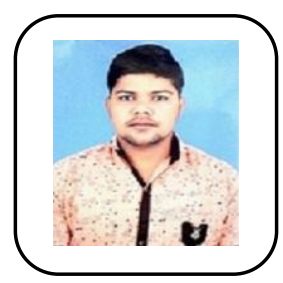

\section{Mr. Vijay Subhash Shinde}

(shindevijay837@gmail.com) is a student of Masters of Technology in Heat Power from School of Mechanical and Civil Engineering, MIT Academy of Engineering Alandi (D), Savitribai Phule Pune University, Pune, India, 412105. He has received the Bachelor of Engineering degree in the department of Mechanical Engineering from NMU University Jalgaon, Maharashtra in the year 2017. His research interest includes Computational Fluid Dynamics, Thermal Engineering. Qualification: Pursuing M. Tech. (Heat Power), MIT Academy of Engineering Alandi (D), Savitribai Phule Pune University, Pune, India, 412105. Mr. Vijay S. Shinde has completed B.E. (Mechanical), SESCOE of Engineering Dhule.

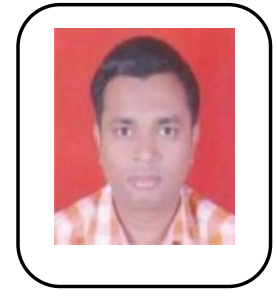

\section{Mr. Pramod P Kothmire}

(ppkothmire@mech.maepune.ac.in), is an Assistant Professor in School of Mechanical and Civil Engineering MIT Academy of Engineering Alandi (D), Savitribai Phule Pune University, Pune, India, 412105. He is student of Ph.D. in Indian Institute of Technology, Bombay, India Since2014 which is joint research program between IITB and MIT Alandi Pune. He has received Master of Technology in Thermal Engineering in Sardar Patel College of Engineering, Andheri, Mumbai India, 2013. He has received Bachelor of Engineering in the department of Mechanical Engineering from Fr. C. Rodrigues Institute of Technology, Vashi, Navi Mumbai, India. 2008. His inter- disciplinary research interests cover Electro hydrodynamics, Interfacial engineering and also HVAC projects. He is passionate to design developed and improves the performance of Oil and refinery industries product. His fore front area of researches is Fluid Mechanics, Heat transfer, Electro hydrodynamics, Interfacial Engineering, Thermal Engineering. He has 7 years of teaching experience, 2.2 year of industrial working experience and 1.2 years of research work. 